\title{
Analytic solutions of the Schrödinger equation with non-central generalized inverse quadratic Yukawa potential
}

\author{
C. O. Edet ${ }^{* 1 @, ~ P . ~ O ~ O k o i ~}{ }^{2}$, S. O Chima ${ }^{2}$ \\ ${ }^{1}$ University of Port Harcourt, Department of Physics, Choba, Nigeria \\ ${ }^{2}$ University of Calabar, Department of Physics, Cross River, Nigeria
}

Received on April 11, 2019. Revised on September 12, 2019. Accepted on September 19, 2019.

\begin{abstract}
This study presents the solutions of Schrödinger equation for the Non-Central Generalized Inverse Quadratic Yukawa Potential within the framework of Nikiforov-Uvarov. The radial and angular part of the Schrödinger equation are obtained using the method of variable separation. More so, the bound states energy eigenvalues and corresponding eigenfunctions are obtained analytically. Numerical results were obtained for the Generalized Inverse Quadratic Yukawa Potential for comparison sake. It was found out that our results agree with existing literature.
\end{abstract}

Keywords: Schrödinger equation; Generalized Inverse Quadratic Yukawa Potential; Non central potential; Nikiforov-Uvarov method; Ring shaped potential.

\section{Introduction}

Physicists over the years have developed strong interest in searching for the solution of the Schrödinger equation with some potentials [1-7]. This is because, finding the analytical solution of the Schrödinger equation is extremely crucial in nonrelativistic quantum mechanics and the eigenfunction contains all the necessary information required to describe a quantum system under consideration.

The analytical solution of the Schrodinger equation with $l=0$ and $l \neq$ for some potentials (central and noncentral) has been addressed by many researchers in nonrelativistic quantum mechanics and relativistic quantum mechanics for bound and scattering states problems[8-16]. Some of the potentials addressed in these studies include; a class of Yukawa potential [17], Coulomb ring-shaped potential [18], ring-shaped Woods-Saxon potential [19], Hartman potential [20-21], Coulombic ring-shaped potential[22] double ring-shaped oscillator potential[23] ringshaped non-spherical harmonic oscillator potential[24-25], spherically harmonic oscillatory ring-shaped potential [26-27], Poschl Teller double-ring-shaped Coulomb potential [28], Manning-Rosen potentials[29-31], inversely quadratic Yukawa potential[32] and Eckart[33]. In most of these studies, the authors used certain well known approximations to overcome the centrifugal barrier $(l \neq 0)$ and obtained the eigenvalues and eigenfunctions using different methods.

The methods which have been employed to solve the differential equation arising from these considerations

\footnotetext{
*Correspondence email address: collinsokonedet@gmail.com
}

include; the asymptotic iteration method (AIM) [34-36], exact quantization rules [37-38], Nikiforov-Uvarov (NU) method [39-41], modified factorization method [42-43], supersymmetric quantum mechanics (SUSYQM) [44-55], and the functional analysis approach (FAA) [46-47].

The generalized inverse quadratic Yukawa potential (GIQYP) was first proposed by [48-49]. This potential is a superposition of the inverse quadratic Yukawa (IQY) [32] and the Yukawa potential [50]. It is asymptotic to a finite value as $r \rightarrow \infty$ and becomes infinite at $r=0$. Oluwadare and Oyewumi [51] solved this potential within the framework of the proper quantization rule and eigenfunction was obtained via the Formula Method [52]. The generalized inverse quadratic Yukawa potential model is of the form [51]

$$
V(r)=-V_{1}\left(1+\frac{e^{-\alpha r}}{r}\right)^{2}
$$

Ikhdair et al. [48] Compared the behaviour of the Yukawatype potential with the Yukawa potential and the IQY potential for screening parameter values, it was noted that differences do not exist between these three potentials.

The Ring shaped potential have a wide range of applications in quantum chemistry and nuclear physics [53]. They have very important role in describing ring-shaped molecules like benzene and the interactions between deformed pair of nuclei [54-55]. They have also been used in demonstrating some of the pseudospin symmetry in nuclei physics [27]. The exact results can be used in 
accounting for some axial symmetric system in quantum chemistry. Zhang et al. [26] proposed a non-central potential as;

$$
V(\theta)=\frac{\hbar^{2}}{2 \mu r^{2}}\left(\frac{C+B \cos ^{2} \theta+A \cos ^{4} \theta}{\sin ^{2} \theta \cos ^{2} \theta}\right)
$$

Motivated by this numerous studies, we attempt to propose a non-central generalized inverse quadratic Yukawa potential (GIQYP) by selecting $V(r)$ as the generalized inverse quadratic Yukawa potential (GIQYP) The noncentral generalized inverse quadratic Yukawa potential (GIQYP) is composed of generalized inverse quadratic Yukawa potential (GIQYP) plus a Novel Angle Dependent (NAD) potential. It can be written as

$$
\begin{aligned}
& V(r, \theta)==-\frac{\eta e^{-2 \alpha r}}{r^{2}}-\frac{\Omega e^{-\alpha r}}{r}-H \\
& +\frac{\hbar^{2}}{2 \mu r^{2}}\left(\frac{C+B \cos ^{2} \theta+A \cos ^{4} \theta}{\sin ^{2} \theta \cos ^{2} \theta}\right)
\end{aligned}
$$

Where $\eta=H=V_{1}$ and $\Omega=2 V_{1}$

The Generalized inverse quadratic Yukawa potential reduces to a constant potential when $\eta=\Omega=0$.

The organization of the work is as follows: In the next section, we give a review of the NU method. In Sect. 3, this method is applied to obtain the bound state solutions with Non-Central Generalized Inverse Quadratic Yukawa Potential. In Sect. 4, we obtain numerical results while in Sect. 5, we give a brief concluding remark.

\section{Review of Nikiforov-Uvarov Method}

The Nikiforov-Uvarov (NU) method is based on solving the hypergeometric-type second-order differential equations by means of the special orthogonal functions [56-57]. The main equation which is closely associated with the method is given in the following form [58];

$$
\psi^{\prime \prime}(z)+\frac{\tilde{\tau}(z)}{\sigma(z)} \psi^{\prime}(z)+\frac{\tilde{\sigma}(z)}{\sigma^{2}(z)} \psi(z)=0
$$

Where $\sigma(z)$ and $\tilde{\sigma}(z)$ are polynomials at most seconddegree, $\tilde{\tau}(z)$ is a first-degree polynomial and $\psi(z)$ is a function of the hypergeometric-type.

The exact solution of Eq. (4) can be obtained by using the transformation

$$
\psi(z)=\phi(z) y(z)
$$

This transformation reduces Eq. (4) into a hypergeometric-type equation of the form

$$
\sigma(z) y^{\prime \prime}(z)+\tau(z) y^{\prime}(z)+\lambda y(z)=0
$$

The function $\phi(z)$ can be defined as the logarithm derivative

$$
\frac{\phi^{\prime}(z)}{\phi(z)}=\frac{\pi(z)}{\sigma(z)}
$$

where

$$
\pi(z)=\frac{1}{2}[\tau(z)-\tilde{\tau}(z)]
$$

with $\pi(z)$ being at most a first-degree polynomial. The second $\psi(z)$ being $y_{n}(z)$ in Eq. (5), is the hypergeometric function with its polynomial solution given by Rodrigues relation

$$
y^{(n)}(z)=\frac{B_{n}}{\rho(z)} \frac{d^{n}}{d s^{n}}\left[\sigma^{n}(z) \rho(z)\right]
$$

Here, $B_{n}$ is the normalization constant and $\rho(z)$ is the weight function which must satisfy the condition

$$
\begin{gathered}
(\sigma(z) \rho(z))^{\prime}=\sigma(z) \tau(z) \\
\tau(z)=\tilde{\tau}(z)+2 \pi(z)
\end{gathered}
$$

It should be noted that the derivative of $\tau(s)$ with respect to $s$ should be negative. The eigenfunctions and eigenvalues can be obtained using the definition of the following function $\pi(s)$ and parameter $\lambda$, respectively:

$$
\begin{aligned}
& \pi(z)=\frac{\sigma^{\prime}(z)-\tilde{\tau}(z)}{2} \\
& \pm \sqrt{\left(\frac{\sigma^{\prime}(z)-\tilde{\tau}(z)}{2}\right)^{2}-\tilde{\sigma}(z)+k \sigma(z)}
\end{aligned}
$$

where

$$
k=\lambda-\pi^{\prime}(z)
$$

The value of $k$ can be obtained by setting the discriminant of the square root in Eq. (12) equal to zero. As such, the new eigenvalue equation can be given as

$$
\lambda_{n}=-n \tau^{\prime}(z)-\frac{n(n-1)}{2} \sigma^{\prime \prime}(z), \quad n=0,1,2, \ldots
$$

\section{Separation of Variables for the Schrodinger Equation}

In spherical coordinates $(r, \theta, \phi)$, the Schrodinger equation with potentials $V(r \theta)$, respectively, can be written as follows[26]:

$$
-\frac{\hbar^{2}}{2 \mu} \nabla^{2} \psi(r, \theta, \phi)+V(r, \theta) \psi(r, \theta, \phi)=E \psi(r, \theta, \phi)
$$

where $E$ is the non-relativistic energy of the system, $\mu$ denotes the rest mass of the particle and $\hbar$ is the planck constant. The Schrodinger equation with potential is given by[54];

$$
\begin{gathered}
{\left[-\frac{\hbar^{2}}{2 \mu}\left[\frac{1}{r^{2}} \frac{\partial}{\partial r} r^{2} \frac{\partial}{\partial r}+\frac{1}{r^{2} \sin \theta} \frac{\partial}{\partial \theta}\left(\sin \theta \frac{\partial}{\partial \theta}\right)\right.\right.} \\
\left.\left.+\frac{1}{r^{2} \sin ^{2} \theta} \frac{\partial^{2}}{\partial \phi^{2}}\right]+V(r, \theta)-E\right] \psi(r, \theta, \phi)=0 \\
\psi(r, \theta, \phi)=R(r) \Theta(\theta) \Phi(\phi)
\end{gathered}
$$




$$
\begin{aligned}
& {\left[-\frac{\hbar^{2}}{2 \mu}\left[\frac{1}{r^{2}} \frac{\partial}{\partial r} r^{2} \frac{\partial}{\partial r}+\frac{1}{r^{2} \sin \theta} \frac{\partial}{\partial \theta}\left(\sin \theta \frac{\partial}{\partial \theta}\right)+\frac{1}{r^{2} \sin ^{2} \theta} \frac{\partial^{2}}{\partial \phi^{2}}\right]+\right.} \\
& \left.\left(-\frac{\eta e^{-2 \alpha r}}{r^{2}}-\frac{\Omega e^{-\alpha r}}{r}-H+\frac{\hbar^{2}}{2 \mu r^{2}}\left(\frac{C+B \cos ^{2} \theta+A \cos ^{4} \theta}{\sin ^{2} \theta \cos ^{2} \theta}\right)\right)-E\right] \psi(r, \theta, \phi)=0
\end{aligned}
$$

Substituting Eq. (17) into Eq. (18) and using the standard procedure of separating variables, we obtain the following differential equations:

$$
\begin{gathered}
\frac{d^{2} R_{n l}}{d r^{2}}+\left[\frac{2 \mu E_{n l}}{\hbar^{2}}-\frac{2 \mu}{\hbar^{2}}\left(-\frac{\eta e^{-2 \alpha r}}{r^{2}}-\frac{\Omega e^{-\alpha r}}{r}-H\right)-\frac{\Lambda}{r^{2}}\right] R_{n l}(r)=0 \\
\frac{d^{2} \Theta(\theta)}{d \theta^{2}}+\frac{\cos \theta}{\sin \theta} \frac{d \Theta(\theta)}{d \theta}+\left(\Lambda-\left(\frac{\hbar^{2}}{2 \mu r^{2}}\left(\frac{C+B \cos ^{2} \theta+A \cos ^{4} \theta}{\sin ^{2} \theta \cos ^{2} \theta}\right)\right)-\frac{m^{2}}{\sin ^{2} \theta}\right) \Theta(\theta)=0 \\
\frac{d^{2} \Phi(\phi)}{d \phi^{2}}+m^{2} \Phi(\phi)=0
\end{gathered}
$$

where $m^{2}$ and $\Lambda$ are separation constants, which are real and dimensionless. The solution of Eq. (21) is periodic and for bound state $\Phi(\phi)$ satisfies the periodic boundary condition $\Phi(\phi+2 \pi)$ and its solutions become,

$$
\Phi(\phi)=\frac{1}{\sqrt{2 \pi}} e^{-i m \phi}, \quad m=0, \pm 1, \pm 2, \ldots
$$

\subsection{Solutions of the radial Schrodinger equation for non-central generalized inverse quadratic Yukawa potential}

$$
\frac{d^{2} R_{n l}}{d r^{2}}+\left[\frac{2 \mu E_{n l}}{\hbar^{2}}-\frac{2 \mu}{\hbar^{2}}\left(-\frac{V_{1} e^{-2 \alpha r}}{r^{2}}-\frac{2 V_{1} e^{-\alpha r}}{r}-V_{1}\right)-\frac{\Lambda}{r^{2}}\right] R_{n l}(r)=0
$$

The radial part of the Schrödinger equation for this potential can be solved exactly for $l=0$ (s-wave) but cannot be solved for this potential for $l \neq 0$. To obtain the solution for $l \neq 0$, we employ the approximation scheme proposed by Greene and Aldrich [59] to deal with the centrifugal term, which is given as;

$$
\frac{1}{r^{2}} \approx \frac{\alpha^{2}}{\left(1-e^{-\alpha r}\right)^{2}}
$$

It is noted that for a short-range potential, the relation (eqs. 24 ) is a good approximation to $\frac{1}{r^{2}}$, as proposed by Greene and Aldrich [59,60-61]. The implies that Eq. (24) is not a good approximation to the centrifugal barrier when the screening parameter $\alpha$ becomes large. Thus, the approximation is valid when $\alpha<<1$. Substituting the approximation (Eq.24) into Eq. 23), we obtain an equation of the form;

$$
\frac{d^{2} R_{n l}}{d r^{2}}+\left[\frac{2 \mu E_{n l}}{\hbar^{2}}-\frac{2 \mu}{\hbar^{2}}\left(-\frac{V_{1} \alpha^{2} e^{-2 \alpha r}}{\left(1-e^{-\alpha r}\right)^{2}}-\frac{2 V_{1} \alpha e^{-\alpha r}}{\left(1-e^{-\alpha r}\right)} V_{1}\right)-\frac{\Lambda \alpha^{2}}{\left(1-e^{-\alpha r}\right)^{2}}\right] R_{n l}(r)=0
$$

Eq. 25p can be simplified into the form and introducing the following dimensionless abbreviations

$$
\left\{\begin{array}{l}
\varepsilon_{n}=\frac{2 \mu\left(E_{n}+V_{1}\right)}{\hbar^{2} \alpha^{2}} \\
\beta=\frac{2 \mu V_{1}}{\hbar^{2}} \\
\chi=\frac{4 \mu V_{1}}{\hbar^{2} \alpha}
\end{array}\right\}
$$

Using a transformation $z=e^{-\alpha r}$ so as to enable us apply the NU method as a solution of the hypergeometric type

$$
\begin{gathered}
\frac{d^{2} R_{n l}(r)}{d r^{2}}=\alpha^{2} z^{2} \frac{d^{2} R_{n l}(z)}{d z^{2}}+\alpha^{2} z \frac{d R_{n l}(z)}{d z} \\
\frac{d^{2} R_{n l}}{d z^{2}}+\frac{(1-z)}{z(1-z)} \frac{d R_{n l}}{d z}+\frac{1}{z^{2}(1-z)^{2}}\left[-\varepsilon_{n}(1-z)^{2}+\beta z^{2}+\chi z(1-\mathrm{z})-\Lambda\right] R_{n l}(z)=0
\end{gathered}
$$


We obtain the differential equation

$$
\frac{d^{2} R_{n l}}{d z^{2}}+\frac{(1-z)}{z(1-z)} \frac{d R_{n l}}{d z}+\frac{1}{z^{2}(1-z)^{2}}\left[-\left(\varepsilon_{n}-\beta+\chi\right) z^{2}+\left(2 \varepsilon_{n}+\chi\right) z-\left(\varepsilon_{n}+\Lambda\right)\right] R_{n l}(z)=0
$$

Comparing Eq. (29) and Eq. (4), we have the following parameters

$$
\left\{\begin{array}{l}
\tilde{\tau}(z)=1-z \\
\sigma(z)=z(1-z) \\
\tilde{\sigma}(s)=-\left(\varepsilon_{n}-\beta+\chi\right) z^{2}+\left(2 \varepsilon_{n}+\chi\right) z-\left(\varepsilon_{n}+\Lambda\right)
\end{array}\right\}
$$

Substituting these polynomials into Eq. 12 , we get $\pi(s)$ to be

$$
\pi(z)=-\frac{z}{2} \pm \sqrt{\left(\frac{1}{4}+\left(\varepsilon_{n}-\beta+\chi\right)-k\right) z^{2}+\left(-\left(2 \varepsilon_{n}+\chi\right)+k\right) z+\left(\varepsilon_{n}+\Lambda\right)}
$$

further rearranged as;

$$
\pi(z)=-\frac{z}{2} \pm \sqrt{\left(\frac{1}{4}+\varepsilon_{n}-\beta+\chi-k\right) z^{2}+\left(k-2 \varepsilon_{n}-\chi\right) z+\left(\varepsilon_{n}+\Lambda\right)}
$$

To find the constant $k$, the discriminant of the expression under the square root of Eq. 313 should be equal to zero. As such, we have that

$$
k_{ \pm}=-(2 \Lambda-\chi) \pm 2 \sqrt{\varepsilon_{n}+\Lambda} \sqrt{\frac{1}{4}+\Lambda-\beta}
$$

Substituting Eq. (33) into Eq. (31) yields

$$
\pi=-\frac{z}{2} \pm\left\{\begin{array}{l}
\left(\sqrt{\eta}_{1}-\sqrt{\eta}_{3}\right) z-\sqrt{\eta}_{1} ; \text { for } k_{+}=-\left(\eta_{2}\right)+2 \sqrt{\eta}_{1} \sqrt{\eta}_{3} \\
\left(\sqrt{\eta}_{1}-\sqrt{\eta}_{3}\right) z+\sqrt{\eta_{1}} ; \text { for } k_{-}=-\left(\eta_{2}\right)-2 \sqrt{\eta}_{1} \sqrt{\eta}_{3}
\end{array}\right\}
$$

where

$$
\left\{\begin{array}{l}
\eta_{1}=\varepsilon_{n}+\Lambda \\
\eta_{2}=2 \Lambda-\chi \\
\eta_{3}=\frac{1}{4}+\Lambda-\beta
\end{array}\right\}
$$

From the knowledge of NU method, we choose the expression $\pi(s)_{-}$which the function $\tau(s)$ has a negative derivative. This is given by

$$
k_{-}=-(2 \Lambda-\chi)-2 \sqrt{\varepsilon_{n}+\Lambda} \sqrt{\frac{1}{4}+\Lambda-\beta}
$$

with $\tau(s)$ being obtained as

$$
\tau(s)=1-2 z-2\left(\sqrt{\varepsilon_{n}+\Lambda}+\sqrt{\frac{1}{4}+\Lambda-\beta}\right) z+2 \sqrt{\varepsilon_{n}+\Lambda}
$$

Referring to Eq. (13), we define the constant $\lambda$ as

$$
\lambda=-(2 \Lambda-\chi)-2 \sqrt{\varepsilon_{n}+\Lambda} \sqrt{\frac{1}{4}+\Lambda-\beta}-\frac{1}{2}-\sqrt{\frac{1}{4}+\Lambda-\beta}-\sqrt{\varepsilon_{n}+\Lambda}
$$

Taking the derivative of $\tau(s)$ from Eq.(37), we have;

$$
\tau^{\prime}(z)=-2-2\left(\sqrt{\varepsilon_{n}+\Lambda}+\sqrt{\frac{1}{4}+\Lambda-\beta}\right)<0
$$

and $\sigma(z)$ from Eq. 30, , we have;

$$
\sigma^{\prime \prime}(z)=-2
$$

Substituting Eq. (39) into Eq. (40), we have

$$
\lambda_{n}=n^{2}+n+2 n \sqrt{\varepsilon_{n}+\Lambda}+2 n \sqrt{\frac{1}{4}+\Lambda-\beta}
$$


Comparing Eq. (38) and (41), and carrying out some algebraic manipulation. We have;

$$
\varepsilon_{n}=-\Lambda+\frac{1}{4}\left[\frac{\left(n+\frac{1}{2}+\sqrt{\frac{1}{4}+\Lambda-\beta}\right)^{2}-\chi+\beta+\Lambda}{\left(n+\frac{1}{2}+\sqrt{\frac{1}{4}+\Lambda-\beta}\right)}\right]^{2}
$$

Substituting Eqs. (17) and Eq. (32) into Eq. (31) yields the energy eigenvalue equation of the Hellman potential in the form

$$
E_{n l}=\frac{\hbar^{2} \alpha^{2} \Lambda}{2 \mu}-V_{1}-\frac{\hbar^{2} \alpha^{2}}{8 \mu}\left[\frac{\left(n+\frac{1}{2}+\sqrt{\frac{1}{4}+\Lambda-\frac{2 \mu V_{1}}{\hbar^{2}}}\right)^{2}-\frac{4 \mu V_{1}}{\hbar^{2} \alpha}+\frac{2 \mu V_{1}}{\hbar^{2}}+\Lambda}{\left(n+\frac{1}{2}+\sqrt{\frac{1}{4}+\Lambda-\frac{2 \mu V_{1}}{\hbar^{2}}}\right)}\right]^{2}
$$

The corresponding wave functions can be evaluated by substituting $\pi(s)_{\text {_ }}$ and $\sigma(s)$ from Eq. (34) and Eq. (30) respectively into Eq. (7) and solving the first order differential equation. This gives

$$
\mathrm{A}(z)=z^{\sqrt{\varepsilon_{n}+\Lambda}}(1-z)^{\frac{1}{2}+\sqrt{\frac{1}{4}+\Lambda-\beta}}
$$

The weight function $\rho(s)$ from Eq. 10 can be obtained as

$$
\rho(z)=z^{2 \sqrt{\varepsilon_{n}+\Lambda}}(1-z)^{2 \sqrt{\frac{1}{4}+\Lambda-\beta}}
$$

From the Rodrigues relation of Eq. (9), we obtain

$$
y_{n}(z) \equiv \Omega_{n, l} P_{n}^{\left(2 \sqrt{\varepsilon_{n}+\Lambda}, 2 \sqrt{\frac{1}{4}+\Lambda-\beta}\right)}(1-2 z)
$$

where $P_{n}^{(\theta, \vartheta)}$ is the Jacobi Polynomial.

Substituting $\mathrm{A}(s) a n d y_{n}(s)$ from Eq. (44) and Eq. (46) respectively into Eq. (5), we obtain the wave function in terms of hyper-geometric polynomial as

$$
R_{n}(z)=\Omega_{n, l} z^{\varpi}(1-z)^{\vartheta} \frac{(2 \omega+1)_{n}}{n !} F_{2} F_{1}(-n, ? 2 \varpi+\vartheta+n ; 2 \varpi+1 ; \mathrm{z})
$$

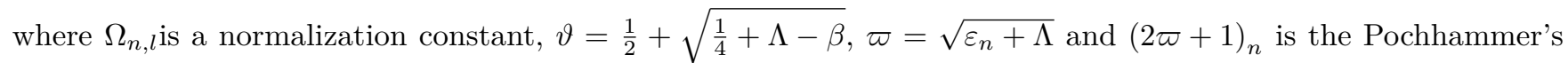
symbol(for the rising factorial)

Using the normalization condition, we obtain the normalization constant as follows [60]:

$$
\begin{gathered}
\int_{0}^{\infty} R_{n, l}(r) \times R_{n, l}(r)^{*} d r=1 \\
-\frac{1}{\alpha} \int_{1}^{0}\left|R_{n, l}(z)\right|^{2} \frac{d z}{z}=1, z=e^{-\alpha r} \\
\frac{1}{2 \alpha} \int_{-1}^{1}\left|R_{n, l}(y)\right|^{2} \frac{2}{1-y} d y=1, y=1-2 z
\end{gathered}
$$

Substituting Eq. (47) into Eq. (50), we have

$$
\frac{\Omega_{n l}^{2}}{2 \alpha} \int_{-1}^{1}\left(\frac{1-y}{2}\right)^{a}\left(\frac{1+y}{2}\right)^{u}\left[P_{n}^{(\mathrm{a}, u-1)}(y)\right]^{2} d y=1
$$

where

$$
u=1+2 \sqrt{\frac{1}{4}+\Lambda-\beta}
$$


and

$$
a=2 \sqrt{\varepsilon_{n}+\Lambda}
$$

Comparing Eq. (51) with the integral of the form [57]

$$
\int_{1}^{-1}\left(\frac{1-p}{2}\right)^{x}\left(\frac{1+p}{2}\right)^{y}\left[P_{n}^{(x, y-1)}(p)\right]^{2} d p=\frac{2 \Gamma(x+n+1) \Gamma(y+n+1)}{\mathrm{n} ! \mathrm{x} \Gamma(x+y+n+1)}
$$

We have the normalization constant as

$$
\Omega_{n l}=\sqrt{\frac{\mathrm{n} ! \mathrm{a} \Gamma(a+u+n+1)}{2 \Gamma(a+n+1) \Gamma(u+n+1)}}
$$

\subsection{Solutions of the angular Schrodinger equation for Non-Central Generalized Inverse Quadratic Yukawa Potential}

In order to get the solution of equation Eq. 200, we introduce a coordinate transformation of the form, $z=\cos ^{2} \theta$ and Eq. 20 becomes

$$
\frac{d^{2} \Theta(z)}{d z^{2}}+\frac{(1-3 z)}{2 z(1-z)} \frac{d \Theta(z)}{d z}+\frac{1}{(2 z(1-z))^{2}}\left(-(\Lambda+B) z^{2}+\left(\Lambda-\mathrm{A}-m^{2}\right) z-C\right) \Theta(z)=0
$$

Similarly, Comparing Eq. (56) and Eq. (4), we have the following parameters

$$
\left\{\begin{array}{l}
\tilde{\tau}(s)=(1-3 z) \\
\sigma(s)=2 z(1-z) \\
\tilde{\sigma}(s)=-(\Lambda+B) z^{2}+\left(\Lambda-\mathrm{A}-m^{2}\right) z-C
\end{array}\right\}
$$

Substituting these polynomials into Eq. (12), we get $\pi(s)$ to be

$$
\pi(z)=-\frac{1-z}{2} \pm \sqrt{\left(\frac{1}{4}+(\Lambda+B)-k\right) z^{2}+\left(-\frac{1}{2}-\left(\Lambda-\mathrm{A}-m^{2}\right)+k\right) z+\frac{1}{4}+C}
$$

Further rearranged as;

$$
\pi(z)=-\frac{1-z}{2} \pm \sqrt{\left(\frac{1}{4}+\Lambda+B-k\right) z^{2}+\left(k-\frac{1}{2}-\Lambda+\mathrm{A}+m^{2}\right) z+\frac{1}{4}+C}
$$

To find the constant $k$, the discriminant of the expression under the square root of Eq. (58) should be equal to zero. As such, we have that

$$
k_{ \pm}=-\frac{\left(\Lambda-\mathrm{A}-m^{2}-C\right)}{2} \pm \frac{1}{2} \sqrt{1+4 C} \sqrt{\mathrm{C}+\mathrm{A}+m^{2}+B}
$$

Substituting Eq. (60) into Eq. (58) yields

$$
\pi=-\frac{z}{2} \pm \frac{1}{2}\left(\left(2 \sqrt{1+4 C}+\sqrt{\mathrm{C}+\mathrm{A}+m^{2}+B}\right) z-2 \sqrt{1+4 C}\right)
$$

From the knowledge of NU method, we choose the expression $\pi(s)$ - which the function $\tau(s)$ has a negative derivative. This is given by

$$
k_{-}=-\frac{\left(\Lambda-\mathrm{A}-m^{2}-C\right)}{2}-\frac{1}{2} \sqrt{1+4 C} \sqrt{\mathrm{C}+\mathrm{A}+m^{2}+B}
$$

with $\tau(s)$ being obtained as

$$
\tau(s)=2-4 z-2\left(\sqrt{1+4 C}+\sqrt{\mathrm{C}+\mathrm{A}+m^{2}+B}\right) z+2 \sqrt{1+4 C}
$$

Referring to Eq. (13), we define the constant $\lambda$ as

$$
\lambda=-\frac{\left(\Lambda-\mathrm{A}-m^{2}-C\right)}{2}-\frac{1}{2} \sqrt{1+4 C} \sqrt{\mathrm{C}+\mathrm{A}+m^{2}+B}-\frac{1}{2}-\frac{1}{2}\left(2 \sqrt{1+4 C}+\sqrt{\mathrm{C}+\mathrm{A}+m^{2}+B}\right)
$$


Taking the derivative of $\tau(s)$ from Eq. 63), we have;

$$
\tau^{\prime}(z)=-4-2\left(\sqrt{1+4 C}+\sqrt{\mathrm{C}+\mathrm{A}+m^{2}+B}\right)
$$

and $\sigma(z)$ from Eq. 57 , we have;

$$
\sigma^{\prime \prime}(z)=-4
$$

Substituting Eq. 65) into Eq. (66), we have

$$
\lambda_{\tilde{n}}=2 \tilde{n}^{2}+2 \tilde{n}+\tilde{n} \sqrt{1+4 C}+\tilde{n} \sqrt{\mathrm{C}+\mathrm{A}+m^{2}+B}
$$

Comparing Eqs (67) and $64\left(\lambda=\lambda_{\tilde{n}}\right)$, and carrying out some algebraic manipulation. We have;

$$
\Lambda=\left(2 \tilde{n}+1+\sqrt{\mathrm{C}+\mathrm{A}+m^{2}+B}\right)^{2}+\sqrt{1+4 C}\left(2 \tilde{n}+1+\sqrt{\mathrm{C}+\mathrm{A}+m^{2}+B}\right)+C-B
$$

or

$$
\Lambda=\left(2 \tilde{n}+1+\sqrt{\mathrm{C}+\mathrm{A}+m^{2}+B}\right)\left(2 \tilde{n}+1+\sqrt{\mathrm{C}+\mathrm{A}+m^{2}+B}+\sqrt{1+4 C}\right)+C-B
$$

The corresponding wave functions can be evaluated by substituting $\pi(s)_{\text {_ }}$ and $\sigma(s)$ from Eq. (57) and Eq. (61) respectively into Eq. (7) and solving the first order differential equation. This gives

$$
\mathrm{E}(z)=z^{\frac{1}{4}+\frac{1}{2} \sqrt{\frac{1}{4}+C}}(1-z)^{\frac{1}{2} \sqrt{\mathrm{C}+\mathrm{A}+m^{2}+B}}
$$

The weight function $\rho(s)$ from Eq. 10 can be obtained as

$$
\rho(z)=z^{\sqrt{\frac{1}{4}+C}}(1-z)^{\sqrt{\mathrm{C}+\mathrm{A}+m^{2}+B}}
$$

From the Rodrigues relation of Eq. (9), we obtain

$$
y_{\tilde{n}}(z) \equiv \chi_{\tilde{n}, m} P_{\tilde{n}}^{\left(\sqrt{\frac{1}{4}+C}, \sqrt{\mathrm{C}+\mathrm{A}+m^{2}+B}\right)}(1-2 z)
$$

where $P_{n}^{(\theta, \vartheta)}$ is the Jacobi Polynomial.

Substituting $\mathrm{E}(s)$ and $y_{\tilde{n}}(s)$ from Eq. (70) and Eq. (72) respectively into Eq. (5), we obtain the wave function in terms of hypergeometric Polynomials as;

$$
\Theta_{\tilde{n} m}(z)=\chi_{\tilde{n}, m} z^{\nu}(1-z)^{\xi} \frac{(2 \nu+1)_{n}}{n !} F_{2}(-n, ? 2 \nu+\xi+n ; 2 \nu+1 ; \mathrm{z})
$$

where $\chi_{\tilde{n}, m}$ is a normalization constant, $\nu=\frac{1}{4}+\frac{1}{2} \sqrt{\frac{1}{4}+C}, \xi=\frac{1}{2} \sqrt{\mathrm{C}+\mathrm{A}+m^{2}+B}$ and $(2 \nu+1)_{n}$ is the Pochhammer's symbol(for the rising factorial)

Now using Eq. 43, we obtain the discrete energy eigenvalues as

$$
\begin{gathered}
E_{n \tilde{n}, m}=\frac{\hbar^{2} \alpha^{2} \kappa}{2 \mu}-V_{0}-\frac{\hbar^{2} \alpha^{2}}{8 \mu}\left[\frac{\left(n+\frac{1}{2}+\sqrt{\frac{1}{4}+\kappa-\frac{2 \mu V_{0}}{\hbar^{2}}}\right)^{2}-\frac{4 \mu V_{0}}{\hbar^{2} \alpha}+\frac{2 \mu V_{0}}{\hbar^{2}}+\kappa}{\left(n+\frac{1}{2}+\sqrt{\frac{1}{4}+\kappa-\frac{2 \mu V_{0}}{\hbar^{2}}}\right)}\right]^{2} \\
\kappa=\left(2 \tilde{n}+1+\sqrt{\mathrm{C}+\mathrm{A}+m^{2}+B}\right)\left(2 \tilde{n}+1+\sqrt{\mathrm{C}+\mathrm{A}+m^{2}+B}+\sqrt{1+4 C}\right)+C-B
\end{gathered}
$$

where $\tilde{n}$ is the number of nodes of the radial wave functions. The $\Lambda$ is the contribution from the angle-dependent part of the potential and plays the role of centrifugal term.

$$
\begin{aligned}
& \psi(r, \theta, \phi)=\frac{N_{\tilde{n} m}}{\sqrt{2 \pi}} z^{\sqrt{\varepsilon_{n}+\Lambda}}(1-z)^{\frac{1}{2}+\sqrt{\frac{1}{4}+\Lambda-\beta}} P_{n}^{\left(2 \sqrt{\varepsilon_{n}+\Lambda}, 2 \sqrt{\frac{1}{4}+\Lambda-\beta}\right)}(1-2 z)\left(\cos ^{2} \theta\right)^{\frac{1}{4}+\frac{1}{2} \sqrt{\frac{1}{4}+C}} \\
& \times\left(\sin ^{2} \theta\right)^{\frac{1}{2} \sqrt{\mathrm{C}+\mathrm{A}+m^{2}+B}} P_{\tilde{n}}^{\left(\sqrt{\frac{1}{4}+C}, \sqrt{\mathrm{C}+\mathrm{A}+m^{2}+B}\right)}(-\cos 2 \theta) e^{-i m \phi}
\end{aligned}
$$

where $\mathrm{N}_{n \tilde{n} m}$ is the new normalization constant. 


\section{Discussion of Numerical Result}

To test the accuracy of our result, we have also computed the energy eigenvalues of the generalized inverse quadratic Yukawa potential using the energy equation given in Eq. 43. . Our results shown in Table S1 are in good agreement with the result of Ref. [51] who solved the Schrodinger Equation with this potential using the Proper Quantization rule within the framework of the Pekeris Approximation scheme. In Figure 1, we plot the behavior of the non-central generalized inverse quadratic Yukawa potential for different values of $r$ and $\theta$.

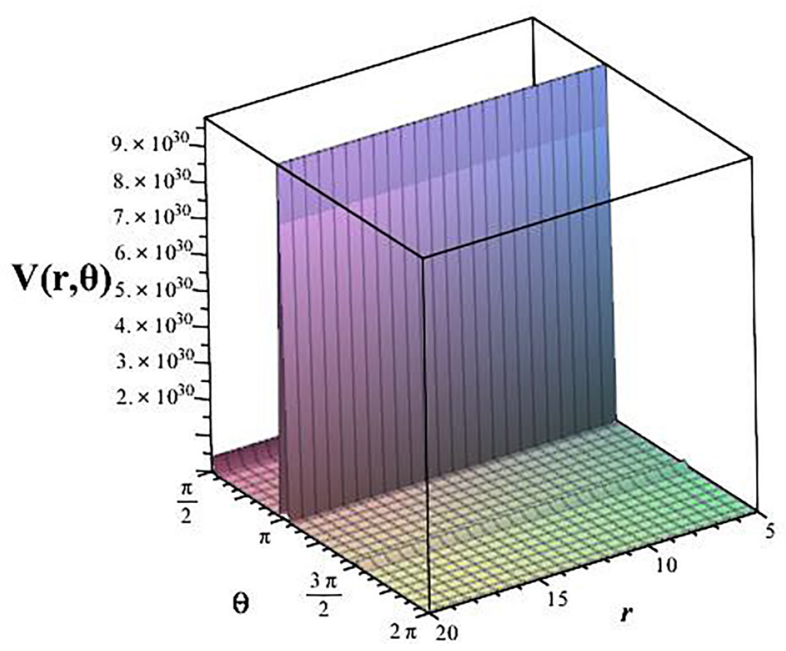

Figure 1: Non-central generalized inverse quadratic Yukawa potential for different values of $r$ and $\theta$.

\section{Conclusions}

We have obtained exact bound states solutions of the three dimensional Schrödinger equation for the NonCentral Generalized Inverse Quadratic Yukawa Potential within the framework of Nikiforov-Uvarov method. The eigenvalues and the corresponding eigenfunctions of the radial and angular parts of the Schrödinger equation was obtained. More so, when the ring shape term vanishes, i.e. $(A=B=C=0)$, then the results are in good agreement with Ref. [51]. Different ring shape potentials can be obtained from this newly proposed Non central potential. Finally, our results can find many applications in nuclear physics and quantum chemistry such as cyclic benzene.

\section{Acknowledgments}

We thank the kind referees for the enlightening comments and suggestions, which have greatly helped us in making improvements to this paper. We appreciate Dr. A. N. Ikot for communicating some of his research materials to us and for continuous encouragement and support. In addition, C. O. Edet dedicates this work to his late father (Mr Okon Edet Udo).

\section{Supplementary material}

The following online material is available for this article:

Table S1. The bound state energy levels (in units of $\mathrm{fm}-1$ ) of the GIQYP for various values of $n, l$ and for $\hbar=\mu=1$

\section{References}

[1] W.C. Qiang, K. Li and W.L. Chen, Journal of Physics A: Mathematical and Theoretical Physics 42, 205306 (2009).

[2] M. Hamzavi, H. Hassanabadi and A.A. Rajabi, Modern Physics Letters A 25, 2447 (2010).

[3] H. Hassanabadi, E. Maghsoodi and S. Zarrinkamar, FewBody System 53, 271 (2012).

[4] H. Hassanabadi, S. Zarrinkamar and A.A. Rajabi, Communications in Theoretical Physics 55, 541 (2011).

[5] H. Hassanabadi, E. Maghsoodi, S. Zarrinkamar and H. Rahimov, Chinese Physics B 21, 120302 (2012).

[6] H. Hassanabadi, S. Zarrinkamar and E. Maghsoodi, Physics Letters B 718, 678 (2012).

[7] H. Hassanabadi, S. Zarrinkamar and E. Maghsoodi, International Journal of Modern Physics A 28, 1350041 (2013)

[8] E. Maghsoodi, H. Hassanabadi, H. Rahimov and S. Zarrinkamar, Chinese Physics C 37, 043105 (2013).

[9] H. Louis, B.I. Ita, O.U. Akakuru, N.A. Nzeata-Ibe, A.I. Ikeuba, T.O. Magu, P.I. Amos and C.O. Edet, Oriental Journal of Physical Sciences 3, 3 (2018).

[10] B.I. Ita, H. Louis, O.U. Akakuru, N.A. Nzeata-Ibe, A.I. Ikeuba, T.O. Magu, P.I. Amos and C.O. Edet, Bulgaria Journal of Physics 45, 323 (2018).

[11] G.F. Wei and S.H. Dong, Physics Letters A 373, 49 (2008).

[12] G.F. Wei and S.H. Dong, The European Physical Journal A 43, 185 (2010).

[13] H. Rahimov, H. Nikoofard, S. Zarrinkamar and H. Hassanabadi, Applied Mathematics and Computation 219, 4710 (2013).

[14] H. Hassanabadi, E. Maghsoodi and S. Zarrinkamar, Commun. Theor. Phys. 58, 807 (2012).

[15] H. Louis, B.I. Ita, T.O. Magu, O.U. Akakuru, N.A. Nzeata-Ibe, A.I Ikeuba, A.I. Pigweh and C.O. Edet, International Journal of Chemistry 10, 99 (2018).

[16] G.F. Wei and S.H. Dong, The European Physical Journal A 46, 207 (2010).

[17] C.A. Onate and J.O. Ojonubah, Journal of Theoretical and Applied Physics 10, 21 (2016).

[18] M.C. Zhang, B. An and H.F. Guo-Qing, Journal Mathematical Chemistry 48, 876 (2010).

[19] H. Hassanabadi, E. Maghsoodi and S. Zarrinkamar, Chinese Physics C 37, 113104 (2013).

[20] C.Y. Chen, Physics Letters A 339, 283 (2005).

[21] A. Souza Dutra and M. Hott, Physics Letters A 356, 215 (2006).

[22] S.H. Dong and M. Lozada-Cassou, Physica Scripta 74, 215 (2006).

[23] F.L. Lu, C.Y. Chen and D.S. Sun, Chinese Physics Letters 14, 463 (2005). 
[24] X.Q. Hu, G. Luo, Z.M. Wu, L.B. Niu and Y. Ma, Communications in Theoretical Physics 53, 242 (2010).

[25] M.C. Zhang, International Journal of Theoretical Physics 48, 2625 (2009).

[26] M.C. Zhang, G.H. Sun and S.H. Dong, Physics Letters A 374, 704 (2010).

[27] B.J. Falaye, Journal of Mathematical Physics 53, 082107 (2012).

[28] E. Maghsoodi, H. Hassanabadi and S. Zarrinkamar, Chinese Physics B 22, 030302 (2013).

[29] B.J. Falaye, K.J. Oyewumi, T.T. Ibrahim, M.A. Punyasena and C.A. Onate, Canadian Journal of Physics 91, 98 (2013).

[30] H. Hassanabadi, E. Maghsoodi, S. Zarrinkamar and H. Rahimov, Canadian Journal of Physics 90, 633 (2012).

[31] H. Hassanabadi, L.L. Lu, S. Zarrinkamar, G. Liu and H. Rahimov, Acta Physica Polinica A 122, 650 (2012).

[32] M. Hamzavi, S.M. Ikhdair and B.I. Ita, Physica Scripta 85, 045009 (2012).

[33] A. Soylu, O. Bayrak and I. Boztosun, Journal of Physics A: Mathematical and Theoretical 41, 065308 (2008).

[34] H. Ciftci, R.L. Hall and N. Saad, Physics Letters A 340, 388 (2005).

[35] H. Ciftci, R.L. Hall and N. Saad, Journal of Physics A: Mathematical and Theoretical 36, 11807 (2003).

[36] B.J. Falaye, Few-Body System 53, 557 (2012).

[37] S.H. Dong, D. Morales and J. García-Ravelo, International Journal of Modern Physics E 16,189 (2007).

[38] S.M. Ikhdair, Journal of Mathematical Chemistry 45, 1137 (2009)

[39] C.O. Edet and P.O. Okoi, Revista Mexicana de Fisica 65, 333 (2019)

[40] C.O. Edet, K.O. Okorie, H. Louis and N.A. Nzeata-Ibe, Indian Journal of Physics (2019).

[41] C.O. Edet, U.S. Okorie, A.T. Ngiangia and A.N. Ikot, Indian Journal Physics (2019).

[42] U.S. Okorie, E.E. Ibekwe, A.N. Ikot, M.C. Onyeaju and E.O. Chukwuocha, Journal of the Korean Physical Society $\mathbf{7 3}, 1211$ (2018).

[43] U.S. Okorie, A.N. Ikot, M.C. Onyeaju and E.O. Chukwuocha, Revista Mexicana de Fisica 64, 608 (2018).
[44] C.A. Onate, M.C. Onyeaju, A.N. Ikot and J.O. Ojonubah, Chinese Journal of Physics 54, 820 (2016).

[45] C.A. Onate, A.N. Ikot, M.C. Onyeaju and M.E. Udoh, Karbala International Journal of Modern Science 3, 1 (2017).

[46] U.S. Okorie, A.N. Ikot, C.O. Edet, I.O. Akpan, R. Sever and R. Rampho, Journal of Physics Communication 3, 095015 (2019).

[47] H.M. Tang, G.C. Liang, L.H. Zhang, F. Zhao and C.S. Jia, Canadian Journal of Chemistry 92, 341 (2014).

[48] S.M. Ikhdair, M. Hamzavi and B.J. Falaye, Applied Mathematics and Computation 225, 775 (2013).

[49] S.M. Ikhdair and M. Hamzavi, Few-Body System 53, 487 (2012).

[50] S.M. Ikhdair, Central European Journal Physics 10, 361 (2012).

[51] O. Oluwadare and K.J. Oyewumi, Chinese Physics Letters 34, 110301 (2017).

[52] B.J. Falaye, S.M. Ikhdair and M. Hamzavi, Few-Body System 56, 63 (2015).

[53] S.H. Dong, G.H. Sun and M. Lozada-Cassou, Physics Letters A 328, 299 (2005).

[54] C. Berkdemir, Journal of Mathematical Chemistry 46, 139 (2009).

[55] C. Berkdemir and Y.F. Cheng, Physica Scripta 79, 034003 (2009).

[56] A.F. Nikiforov and V.B. Uvarov, Special functions of Mathematical Physics (Birkhäuser Basel, Boston, 1988).

[57] O. Ebomwonyi, C.A. Onate, M.C. Onyeaju and A.N. Ikot, Karbala International Journal of Modern Science 3, 59 (2017).

[58] G. Sezgo, Orthogonal Polynomials (American Mathematical Society, New York, 1939).

[59] R.L. Greene and C. Aldrich, Physical Revew A 14, 2363 (1976).

[60] M.R. Setare and S. Haidari, Physica Scripta 18, 065201 (2010)

[61] W.C. Qiang, K. Li and W.L. Chen, Journal of Physics A: Mathematical and Theoretical 42, 205306 (2009). 